INPLASY

PROTOCOL

To cite: Dong et al. Lapatinib with whole brain radiotherapy in breast cancer patients with brain metastases: study protocol of a systematic review and pooled analysis. Inplasy protocol 2020120089. doi: 10.37766/inplasy2020.12.0089

Received: 16 December 2020

Published: 17 December 2020

Corresponding author:

Fan Fei

lovespiderman@qq.com

Author Affiliation:

Sichuan Academy of Medical Sciences and Sichuan

Provincial People's Hospital; School of Medicine, University of Electronic Science

Support: Sichuan Science and Technology.

Review Stage at time of this submission: The review has not yet started.

\section{Lapatinib with whole brain radiotherapy in breast cancer patients with brain metastases: study protocol of a systematic review and pooled analysis}

Dong, WT¹; Xu, RX2; Liao, XL³; He, YS4; Hao, L5; He, ZZ6; Fei, F7.

Review question / Objective: Evaluate the efficacy and safety of lapatinib with whole brain radiotherapy for the treatment of breast cancer patients with brain metastases. P: breast cancer patients with brain metastases. I: Patients treated with whole brain radiotherapy (WBRT) in combination with lapatinib. C: Patients treated with whole brain radiotherapy (WBRT) or lapatinib alone. O: The primary outcome is central nervous system (CNS) objective response rate (ORR). CNS objective responses were defined as either a complete response (CR) or partial response (PR), assessed by volumetric analyses by magnetic resonance imaging (MRI). The second outcome are overall survival (OS), time to progression (TTP), safety and tolerability. S: Randomized controlled trial.

INPLASY registration number: This protocol was registered with the International Platform of Registered Systematic Review and Meta-Analysis Protocols (INPLASY) on 17 December 2020 and was last updated on 29 December 2020 (registration number INPLASY2020120089).

Conflicts of interest:

None.

\section{INTRODUCTION}

Review question / Objective: Evaluate the efficacy and safety of lapatinib with whole brain radiotherapy for the treatment of breast cancer patients with brain metastases. P: breast cancer patients with brain metastases. I: Patients treated with whole brain radiotherapy (WBRT) in combination with lapatinib. C: Patients treated with whole brain radiotherapy (WBRT) or lapatinib alone. O: The primary 
outcome is central nervous system (CNS) objective response rate (ORR). CNS objective responses were defined as either a complete response (CR) or partial response (PR), assessed by volumetric analyses by magnetic resonance imaging (MRI). The second outcome are overall survival (OS), time to progression (TTP), safety and tolerability. S: Randomized controlled trial.

Condition being studied: Breast cancer (BC) is one of the common tumors that cause brain metastasis (BMs). With improvements in overall survival (OS), the probability of brain metastasis is relatively increasing in BC patients. Previous studies demonstrated that BMs may be diagnosed in $10-30 \%$ of cases in advanced BC patients, especially those with human epidermal growth factor receptor (HER-2) positive. Options to the treatment of BMs include surgery and stereotactic radiosurgery (SRS) and whole brain radiation therapy (WBRT). Systemic anticancer treatment is not generally used in BMs since the blood-brain barrier (BBB) prevents therapeutic agents from entering the central nervous system (CNS). Lapatinib, that inhibits both EGFR and HER-2 targets, has a small molecular size and a high BBB penetrability and is approved for the treatment of her-2 positive metastatic BC. Also, WBRT is an option to treat $B M s$ and it may be helpful to open the BBB to elevate the concentration of Lapatinib in CNS to provide synergistic activity of lapatinib and WBRT. To date there has been little agreement on the efficacy and safety of lapatinib combine with WBRT. Therefore, the aim of this metaanalysis was to evaluate the efficacy and safety of lapatinib with WBRT in BC patients with BMs.

\section{METHODS}

Participant or population: Inclusion criteria: (1) Patients pathologically diagnosed as breast cancer; (2) Patients with at least one measurable lesion in the brain, defined as any lesion of $\geq 10 \mathrm{~mm}$ in the longest dimension on T1-weighted, gadoliniumenhanced MRI; (3) Normal organ and marrow function. Exclusion criteria: (1) BC patients synchronous with other malignancies; (2) Patients suitable for brain surgery or stereotactic radiosurgery (SRS); (3) Patients who had prior cranial radiotherapy or SRS (4) Patients with leptomeningeal carcinomatosis as the only site of CNS involvement (5) Pregnant and lactating women.

Intervention: The treatment group will receive whole brain radiotherapy (WBRT) in combination with lapatinib.

Comparator: The control group will receive whole brain radiotherapy (WBRT) or lapatinib alone.

Study designs to be included: Published randomized controlled trials.

Eligibility criteria: Inclusion criteria: (1) Patients pathologically diagnosed as breast cancer; (2) Patients with at least one measurable lesion in the brain, defined as any lesion of $\geq 10 \mathrm{~mm}$ in the longest dimension on T1-weighted, gadoliniumenhanced MRI; (3) Normal organ and marrow function.

Information sources: Searches on databases PubMed, Cochrane and Embase were performed in June, 2020 without filters of date or language. PubMed And Cochrane The descriptors and free term of breast cancer include Breast Neoplasm, Neoplasm, Breast, Breast Tumors, Breast Tumor, Tumor, Breast, Tumors, Breast, Breast Cancer, Mammary Cancer, Malignant Neoplasm of Breast, Breast Malignant Neoplasm, Malignant Tumor of Breast, Breast Malignant Tumor, Cancer of Breast, Human Mammary Carcinomas, Breast Carcinoma, Breast Carcinomas. The descriptors and free term of brain include Brain, Encephalon. The descriptors and free term of metastasis include Neoplasm Metastasis, Metastases, Neoplasm Metastasis, Metastasis, Neoplasm, Neoplasm Metastases. The descriptors and free term of lapatinib include Lapatinib, Lapatinib Ditosylate. The descriptors and free term of whole brain radiotherapy include whole brain radiotherapy, WBRI. 
Main outcome(s): The primary outcome is central nervous system (CNS) objective response rate (ORR). CNS objective responses were defined as either a complete response (CR) or partial response (PR), assessed by volumetric analyses by magnetic resonance imaging (MRI).

Quality assessment / Risk of bias analysis: The risk of bias will be assessed using the Cochrane Collaboration's tool for randomized trials.

Strategy of data synthesis: In this metaanalysis, a quantitative synthesis and aggregate participant data will be used to evaluate the efficacy and safety of lapatinib with whole brain radiotherapy for the treatment of breast cancer patients with brain metastases. Results, such as the central nervous system (CNS) objective response rate (ORR), overall survival (OS), time to progression (TTP) and adverse events will be reported as risk ratios (RRs) with their $95 \%$ confidence intervals (Cls). The mean difference(MD) and the 95\% credible intervals $(\mathrm{Cl})$ will be calculated for the continuous variable. For dichotomous data, treatment effects were expressed as odds ratio (OR). $95 \%$ credible intervals (Crls) which could be interpreted as $95 \% \mathrm{CI}$ were used for the estimating the efficacy and safety of lapatinib with whole brain radiotherapy in breast cancer patients with brain metastases. The meta analysis will be performed in R, version 3.3.3 and Stata, version 12 with the Bayesian methods and fixed-effect model. We used the node-split method to assess inconsistency between indirect and direct comparisons in closed loops, and a P-value lower than 0.05 indicates a statistically significant inconsistency. The consistency model was used when there was no significant inconsistency; otherwise, the inconsistency model was conducted. All discrepancies will be settled by negotiation between the two researchers.

Subgroup analysis: Subgroup analyses will investigate the effects of lapatinib with whole brain radiotherapy according to the dose of lapatinib and the Gy fractions of whole brain radiotherapy.
Sensibility analysis: Sensitivity analysis will be carried out based on the sample size, the missing data result and the methodological quality of the included study. If necessary, we will exclude a lowquality study and repeat the meta-analysis to test the stability of the pooled results.

\section{Country(ies) involved: China.}

Keywords: Breast cancer; Brain metastasis; Whole brain radiation therapy; Lapatinib; Meta-analysis.

Contributions of each author:

Author 1 - Wentao Dong.

Author 2 - Ruxiang Xu.

Author 3 - Xiaoling Liao.

Author 4 - Yongsheng He.

Author 5 - Lina Hao.

Author 6 - Zongze He.

Author 7 - Fan Fei. 